\title{
FOLFOX vs FOLFIRI as Second-line of Therapy After Progression to Gemcitabine/Nab-paclitaxel in Patients with Metastatic Pancreatic Cancer
}

This article was published in the following Dove Press journal: Cancer Management and Research

\author{
Martina Catalano' \\ Raffaele Conca ${ }^{2}$ \\ Roberto Petrioli ${ }^{3}$ \\ Monica Ramello ${ }^{4}$ \\ Giandomenico Roviello (1D ${ }^{5}$ \\ 'School of Human Health Sciences, \\ University of Florence, Florence 50134, \\ Italy; ${ }^{2}$ Division of Medical Oncology, \\ Department of Onco-Hematology, \\ IRCCS-CROB, Referral Cancer Center of \\ Basilicata, Rionero, Vulture (PZ) 85028, \\ Italy; ${ }^{3}$ Department of Medicine, Surgery \\ and Neurosciences, Medical Oncology \\ Unit, University of Siena, Siena 53100, \\ Italy; ${ }^{4}$ Oncology Unit, Department of \\ Medical, Surgical, \& Health Sciences, \\ University of Trieste, Trieste, Italy; \\ ${ }^{5}$ Department of Health Sciences, \\ University of Florence, Florence 50I39, \\ Italy
}

Background: Several progresses have been achieved for first-line chemotherapy in metastatic pancreatic ductal adenocarcinoma (PDAC) with Gem-NabP and FOLFIRINOX extensively used as standard first line regimens. However, the best second-line chemotherapy choice after progression is still not completely defined. The aim of this study is to compare effectiveness and safety of two possible second-line therapeutic options, FOLFOX and FOLFIRI, after progression to Gem-NabP.

Methods: From January 2015 to December 2018, patients with metastatic PDAC, progressed to the first-line treatment with Gem-NabP, and treated with a fluoropyrimidine-based second-line chemotherapy were considered eligible for our retrospective analysis. Overall survival (OS) and progression free survival (PFS) were set as primary endpoints whereas, disease control rate (DCR) and the rate and severity of treatment-related AEs were secondary endpoints.

Results: Overall, 31 patients were treated with Gem-NabP in first-line regimen, 11 received second-line with FOLFOX and 20 with FOLFIRI after progression. Baseline demographic and clinic features were similar in the two groups excluding median age of 55.5 years (range: 50-73) and 68 years (range: 59-72) in FOLFIRI and FOLFOX groups, respectively $(p=0.002)$. Median PFS was three months $(95 \% \mathrm{CI}: 3-4)$, with no significative difference between the two groups. Median OS was eight months (95\%CI: 5-10) and was significantly higher in the FOLFIRI group compared with the FOLFOX group, nine months (95\%CI: 7-17) vs five months (95\%CI: $2-10 ; p<0.01)$. The most commonly reported adverse events were grade 1 or 2 with anemia most frequent in the FOLFOX group (36.4\% vs $10.0 \%)$ and diarrhea in the FOLFIRI group (40.0\% vs 9.1\%). Grade $3-4$ adverse events as neutropenia, diarrhea and nausea/vomiting, occurred in 10 patients $(32.2 \%)$ without differences between the two groups.

Conclusion: Our results seem to support the use of fluoropyrimidine-based second-line therapy for patients with metastatic pancreatic cancer, confirming the effectiveness and safety, to a greater extent with FOLFIRI regimen, after progression to the Gem-NabP.

Keywords: irinotecan, oxaliplatin, second line, pancreatic cancer

\section{Introduction}

Pancreatic adenocarcinoma is an aggressive disease characterized by a very poor prognosis and represents the fourth leading cause of cancer-related deaths in Europe and US. ${ }^{1,2}$ In recent years, progress has been made regarding the first-line of chemotherapy in locally advanced and metastatic pancreatic ductal adenocarcinoma (PDAC). These therapies are based on a combination of four drugs: 5-fluorouracil,
Correspondence: Giandomenico Roviello Department of Health Sciences, University of Florence, Viale Pieraccini, 6, Florence 50139 , Italy

Tel $+055-79383$ I 3

Email giandomenicoroviello@hotmail.it 
oxaliplatin, irinotecan, and folinic acid as in FOLFIRINOX regimen or on combination of two drugs such as gemcitabine and nab-paclitaxel (GemNabP). ${ }^{3,4}$ In general, although FOLFIRINOX has been usually reserved for younger patients with good performance status, no standard first-line of treatment has been validated and choices are made based on local guidelines of medical preference. In addition, the increase of options for second-line chemotherapy, questions on treatment and sequence choice have arisen. Currently, a second-line based on fluoropyrimidine (FP) alone or in combination with oxaliplatin (L-OHP) or irinotecan, after progression from the first-line therapy containing gemcitabine, is recommended by America Society of Clinical Oncology (ASCO). ${ }^{5}$ Recently, based on the results of the NAPOLI-1 trial, the Food and Drug Administration (FDA) approved combination of nano-liposomal-IRI (Nal-IRI) with 5-FU and folinic acid in previously treated patients with gemcitabine-based chemotherapy. ${ }^{6}$ Moreover, studies testing FOLFIRINOX after Gem-NabP failure are ongoing. Thus, the opportunity to choose various 5-FUbased regimens (ie, FOLFOX, FOLFIRI, nal-IRI or FOLFIRINOX) as second-line treatment, seems advantageous after the first-line use of Gem-NabP. However, no randomized trials indicate the sequence to achieve the best efficacy and safety. The aim of this retrospective study is to compare two of the possible second-line therapeutic options, after progression to the first-line treatment with Gem-NabP.

\section{Methods}

\section{Study Population}

Patients of this study derive from the multicenter observational retrospective study NAPA. In the NAPA study, we retrospectively evaluated patients with metastatic PDAC treated with first-line Gem-NabP according clinical guidelines from four Italian oncological units between January 2015 to December 2018 (data not yet reported). Inclusion criteria were age $\geq 18$ years, histopathological confirmed PDAC, Eastern Cooperative Oncology Group Performance Status (ECOG-PS) 0-1, satisfactory hematological function (neutrophil count $\geq 1500 / \mathrm{mm}^{3}$, platelet count $\geq 100,000 / \mathrm{mm}^{3}$, and hemoglobin $\geq 9 \mathrm{~g} / \mathrm{dL}$ ), biochemical blood profiles and glomerular filtration rate. Patients treated with fluoropyrimidine-free second-line regimen, with locally advanced cancer, who received secondary chemoradiotherapy (CRT) treatment or with other histology than PDAC were excluded. Written informed consent was obtained from each patient before starting treatment (trial registration number: 14565_oss; Comitato Etico Regionale per la Sperimentazione Clinica della Regione Toscana: Sezione: AREA VASTA CENTRO; Careggi University of Florence Hospital Ethical Committee). In this study, we report the retrospective analysis on the efficacy and safety of two different fluoropyrimidine-based second-line chemotherapy treatments. The choice of second-line chemotherapy was decided locally in each center.

\section{Treatment and Outcomes}

The second-line regimen with FOLFOX or FOLFIRI was administered until disease progression, unacceptable toxicity or patient refusal. FOLFOX was administered every two weeks (L-OHP on day 1 at the dose of $85 \mathrm{mg} / \mathrm{m}^{2}$ as a two-hour infusion, concurrently with iv LV $200 \mathrm{mg} / \mathrm{m}^{2}$ / day followed by bolus 5-FU $400 \mathrm{mg} / \mathrm{m}^{2}$ and 5-FU $600 \mathrm{mg} /$ $\mathrm{m}^{2}$ continuous infusion for two consecutive days). FOLFIRI was administered every two weeks (irinotecan $150 \mathrm{mg} / \mathrm{m}^{2}$ as a one-hour infusion on day 1 , folinic acid $100 \mathrm{mg} / \mathrm{m}^{2}$ intravenously on days $1-2$, and 5-fluorouracil as a $400 \mathrm{mg} / \mathrm{m}^{2}$ bolus and then as a $600 \mathrm{mg} / \mathrm{m}^{2}$ continuous infusion over $22 \mathrm{~h}$ on days $1-2$ ). Tumor response evaluation was performed every 12 weeks by chest-abdomen computed tomography (CT). The best response during second-line treatment was radiologically evaluated according to the Response Evaluation Criteria in Solid Tumors (RECIST) version 1.1. ${ }^{7}$ Furthermore, measurement of the carbohydrate antigen (CA)19-9 serum level was performed in addiction to the radiological evaluation every 12 weeks. Blood tests were performed at baseline and every cycle. All treatment-related adverse events (AEs) were recorded according to the National Cancer Institute Common Terminology Criteria for Adverse Events (NCI-CTCAE) version $4.0{ }^{8}$

\section{Statistical Analysis}

The primary endpoint was overall survival (OS), the secondary endpoints were disease control rate (DCR); and progression free survival (PFS) and the rate and severity of treatment-related AEs. Overall survival was defined as a time from the start to the second-line treatment to death from any cause. PFS was defined as time from the start date of the second-line to the date of first progression in second-line treatment. Disease control rates are defined as the percentage of patients who have 
achieved complete response (CR), partial response (PR) and stable disease. Stable disease was the best overall response in our cohort. No complete or partial responses were recorded in our sample. PFS and OS were determined using the Kaplan-Meier method to provide the median value and $95 \% \mathrm{CI}$ and treatment groups were compared using the log rank test. Baseline clinical characteristics, response rates, and AEs were compared using chi-squared statistics. All data were analyzed by STATA software.

\section{Results}

\section{Patient and Tumor Characteristics}

Between January 2015 and January 2018, 115 patients with metastatic pancreatic cancer treated in first-line with Gem-NabP were identified. Forty-seven (40.9\%), received second-line therapy, of these 31 with fluoropyrimidine and then they were included in this study (Table 1). Eleven patients received FOLFOX treatment and 20 patients FOLFIRI treatment. Median age was 55.5 years (range: $50-73$ ) with four (20\%) patients over 65 years in the FOLFIRI group, and 68 years (range: 59-72) with seven $(63.6 \%)$ patients over 65 years in the FOLFOX group $(p=0.002)$ with a comparable male:female ratio. ECOG-PS was 0 in the $51.6 \%$ of patients and one in the $48.4 \%$ without significant differences. The more frequent metastatic sites were liver, lungs, and peritoneum; 19 had one metastatic site $(61.3 \%)$ and $13(41.9 \%)$ three or more, statistically comparable between the two groups. Patients who received the FOLFIRI regimen, have been treated with surgery and biliary stent in $12.9 \%$ and $22.6 \%$ respectively, and radiation therapy has been performed in one patient. Among previous surgeries, two patients underwent to duodenopancreatectomy, and two underwent distal pancreatectomy. No previous treatment in the FOLFOX group was performed. About $70 \%$ of the patients in the two groups had received more than four cycles of Gem-NabP. Median baseline CA 19.9 was $934 \mathrm{U} / \mathrm{mL}$ (range: 14-13,027 U/mL) higher in FOLFOX group than FOLFIRI (1523 U/mL vs $450 \mathrm{U} / \mathrm{mL})$. Four patients $(36.4 \%)$ in the FOLFOX group and $12(60 \%)$ in the FOLFIRI group reported cancer-related pain before treatment. Median PFS was six months. In $16(80 \%)$ and six (54.4\%) patients in the FOLFIRI and FOLFOX group respectively a PFS $>3$ months with Gem-NabP was reached. A total of 14 patients performed a third-line of
Table I Patient Characteristics

\begin{tabular}{|c|c|c|c|}
\hline & $\begin{array}{l}\text { Overall } \\
(N=31)\end{array}$ & $\begin{array}{l}\text { FOLFOX } \\
(\mathrm{N}=11)\end{array}$ & $\begin{array}{l}\text { FOLFIRI } \\
(\mathbf{N}=\mathbf{2 0})\end{array}$ \\
\hline \multicolumn{4}{|l|}{ Age, years } \\
\hline Median & 59 & 68 & 55.5 \\
\hline Range & $50-73$ & $59-72$ & $50-73$ \\
\hline$\geq 65$ & II (35.5\%) & $7(63,6 \%)$ & $4(20 \%)$ \\
\hline \multicolumn{4}{|l|}{ ECOG-PS } \\
\hline 0 & $16(51.6 \%)$ & $7(63.6 \%)$ & $9(45 \%)$ \\
\hline 1 & 15 (48.4\%) & $4(36.4 \%)$ & II (55\%) \\
\hline \multicolumn{4}{|l|}{ Sex } \\
\hline Male & 19 (61.3\%) & $5(45.5 \%)$ & $14(70 \%)$ \\
\hline Female & $12(38.7 \%)$ & $6(54.5 \%)$ & $6(30 \%)$ \\
\hline \multicolumn{4}{|l|}{ Site of metastatic disease } \\
\hline Liver & $19(61.3 \%)$ & $6(54.5 \%)$ & $13(65 \%)$ \\
\hline Lung & $6(19.3 \%)$ & $3(27.2 \%)$ & $3(15 \%)$ \\
\hline Peritoneum & $4(12.9 \%)$ & $2(18.2 \%)$ & $2(10 \%)$ \\
\hline Others & $2(6.4 \%)$ & I (9.1\%) & I (5\%) \\
\hline \multicolumn{4}{|l|}{$\begin{array}{l}\text { Number of metastatic } \\
\text { sites }\end{array}$} \\
\hline $1-2$ & $19(61.3 \%)$ & $6(54.5 \%)$ & $12(60 \%)$ \\
\hline$\geq 3$ & $13(41.9 \%)$ & 5 (45.5\%) & $8(40 \%)$ \\
\hline \multicolumn{4}{|l|}{$\begin{array}{l}\text { Carbohydrate antigen } \\
19-9-\mathrm{U} / \mathrm{mL}^{\mathrm{a}}\end{array}$} \\
\hline Median & 934 & 1523 & 450 \\
\hline Range & $14-13,027$ & $521-7295$ & $14-13,027$ \\
\hline \multicolumn{4}{|l|}{ Previous treatment } \\
\hline Radiation therapy & I (3.2\%) & 0 & I (5\%) \\
\hline Surgery & $4(12.9 \%)$ & 0 & $4(20 \%)$ \\
\hline Biliary stent & 7 (22.6\%) & 0 & $7(35 \%)$ \\
\hline \multicolumn{4}{|l|}{ Pain } \\
\hline Yes & $16(51.6 \%)$ & $4(36.4 \%)$ & $12(60 \%)$ \\
\hline \multicolumn{4}{|l|}{ Previous NabGem } \\
\hline Median PFS months $(95 \% \mathrm{Cl})$ & $6(4-7)$ & $5(3-5)$ & $6(4-8)$ \\
\hline PFS $>3$ months & 22 (7|\%) & $6(54.5 \%)$ & $16(80 \%)$ \\
\hline$>4$ cycles of $\mathrm{NabGem}$ & 7 (7।\%) & $6(54.5 \%)$ & $\mathrm{I}(15 \%)$ \\
\hline
\end{tabular}

Note: a Unit of measure.

therapy (nine in the FOLFIRI group and five in the FOLFOX group).

\section{Efficacy}

Stable disease and disease progression were recorded in $12.9 \%$ and $64.5 \%$ of patients respectively, with no difference between the two groups. Response was not evaluable for seven patients. Median PFS was three months (95\%CI: 3-4), with no difference between the two groups (Figure 1). Median OS was eight months in all patients $(95 \% \mathrm{CI}$ : 5-10) and was significantly higher in the FOLFIRI group 


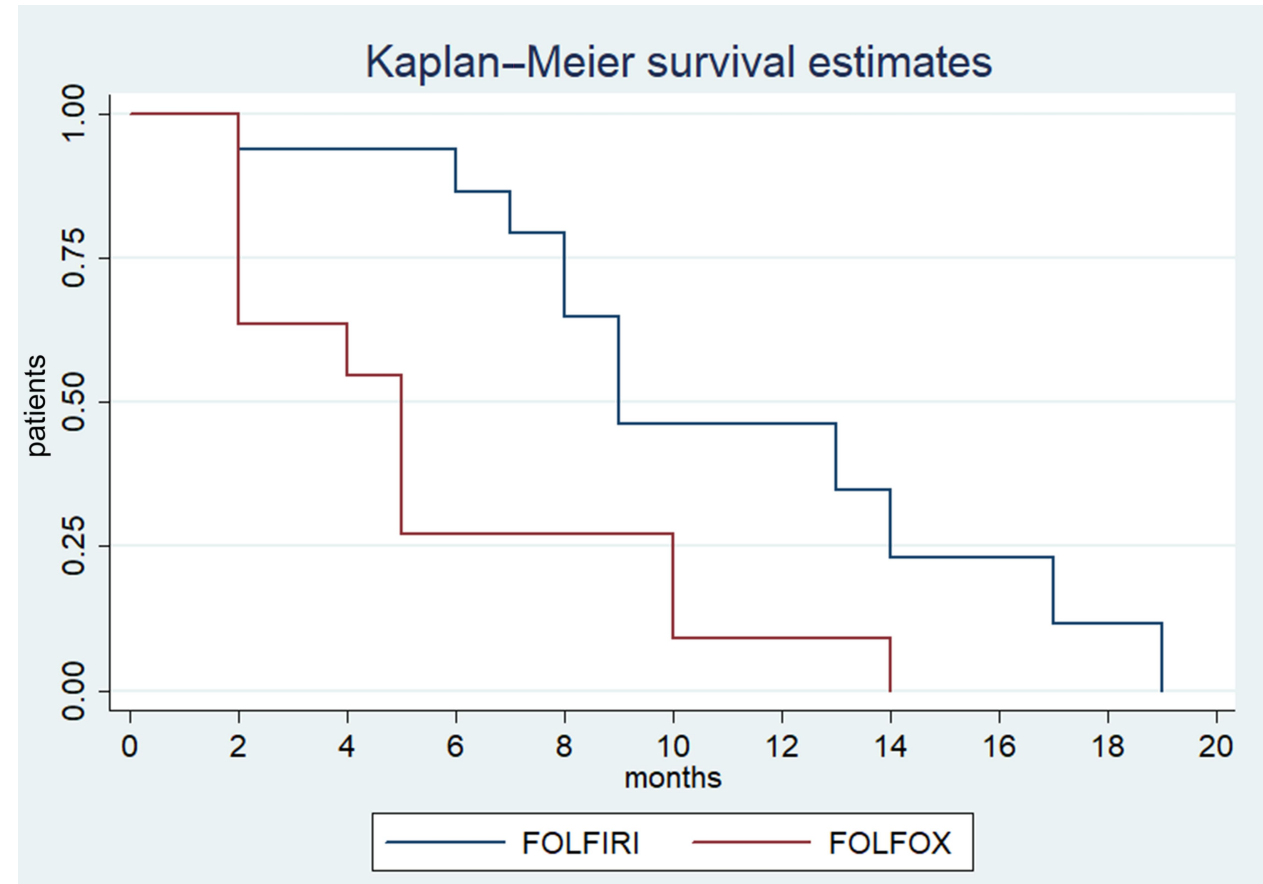

Figure I Estimated OS for FOLFOX compared with FOLFIRI.

(nine months; 95\%CI: 7-17) compared with the FOLFOX group (five months; 95\%CI: 2-10; $p<0.01$ ) (Figure 1). Moreover, median OS was significantly higher in the FOLFIRI group (nine months; 95\%CI: 7-17) compared with the FOLFOX group (two months; 95\%CI: two not reached; $p<0.02$ ) for patients in which PFS $>3$ months with Gem-NabP was recorded (Figure 2). In the univariate analysis, number of metastatic sites $\geq 3$ was negatively

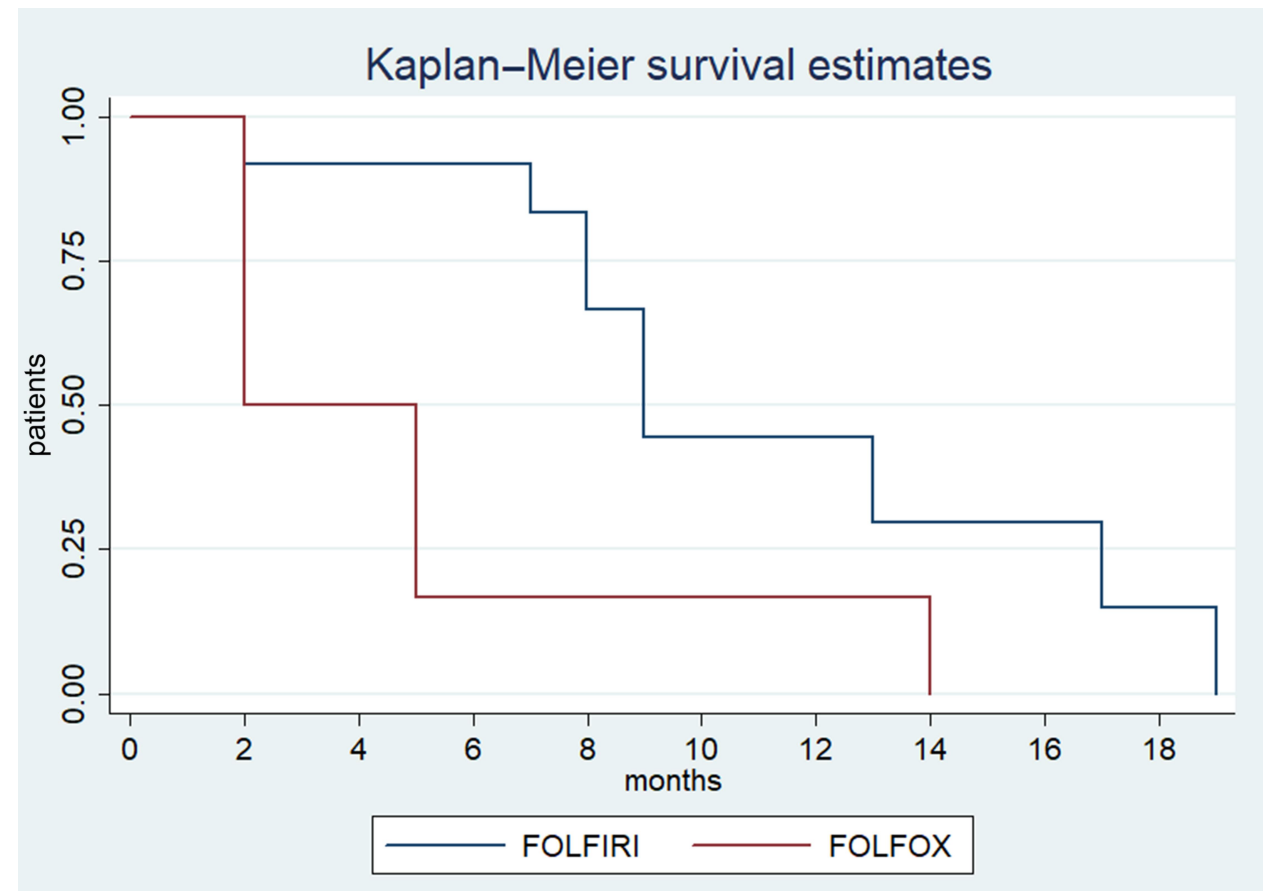

Figure 2 Estimated OS for FOLFOX compared with FOLFIRI for patients in which PFS >3 months with Gem-NabP. 
Table 2 Best Response, PFS and OS According to Neutropenia Grade

\begin{tabular}{|c|c|c|c|c|}
\hline & Overall $(\mathbf{N}=\mathbf{3} I)$ & FOLFOX $(\mathbf{N}=I I)$ & FOLFIRI $(\mathbf{N}=20)$ & $\mathbf{P}$ \\
\hline Stable disease & $4(12.9 \%)$ & I (9.1\%) & $3(15 \%)$ & 0.35 \\
\hline Progression of disease & $20(64.5 \%)$ & $10(90.9 \%)$ & $10(50 \%)$ & \\
\hline Not evaluated & 7 (22.6\%) & 0 & $7(35 \%)$ & \\
\hline PFS M-months $(95 \% \mathrm{Cl})$ & $3(3-4)$ & $2(2-4)$ & $3(3-N R)$ & 0.14 \\
\hline \multicolumn{5}{|l|}{ OS M-months $95 \% \mathrm{Cl}$} \\
\hline All patients & $8(5-10)$ & $5(2-10)$ & $9(7-17)$ & $0.01 *$ \\
\hline Patients with PFS $>3$ months with NabGem & $8(4-8)$ & $2(2-N R)$ & $9(7-17)$ & $0.02 *$ \\
\hline
\end{tabular}

Note: *statistically significant.

Abbreviations: M, median; PFS, progression free survival; OS, overall survival; NR, not reached).

correlated with OS (HR: 3.49 ; 95\%CI: 1.91-5.48; $p=0.01$ ). The FOLFIRI regimen was correlated with better OS compared to the FOLFOX regimen (HR: 0.35 ; $95 \% \mathrm{CI}: 0.14$ $0.87 ; p=0.02)$. Other variables examined have resulted not significant (Table 2). Performing a multivariate analysis, number of metastatic sites $\geq 3$ was confirmed as an independent and negative prognostic indicator for OS (HR: 3.01; 95\%CI: 1.85-4.24; $p=0.02$ ). In contrast, treatment with FOLFIRI was again correlated with a better OS than FOLFOX (HR: 0.66; 95\%CI: 0.45-0.72; $p=0.04$ ) (Tables 3 and 4).

\section{Safety}

Hematological and nonhematological AEs are shown in Table 5. The most common toxicities were grade 1 or 2 : anemia, recorded in four $(36.4 \%)$ patients treated with FOLFOX and in two (10\%) patients with FOLFIRI; conversely, diarrhea was most frequent in the FOLFIRI group than FOLFOX, experienced by five (40\%) and one $(9.1 \%)$ patients, respectively. Grade 3-4 AEs occurred in 10

Table 3 Univariate Analysis for OS

\begin{tabular}{|c|c|c|c|}
\hline & HR & $95 \% \mathrm{Cl}$ & $P$ \\
\hline Age $\geq 65$ & 1.47 & $0.60-3.60$ & 0.39 \\
\hline ECOG-PS (I vs 0 ) & 0.98 & $0.4 I-2.35$ & 0.98 \\
\hline Sex (male vs female) & 0.92 & $0.38 I-2.21$ & 0.85 \\
\hline No. of metastatic sites $\geq 3$ & 3.49 & $1.9 I-5.48$ & $0.01 *$ \\
\hline Carbohydrate antigen $19-9 \geq 600 \mathrm{U} / \mathrm{mL}$ & 1.44 & $0.57-3.61$ & 0.43 \\
\hline Previous radiation therapy & 0.37 & $0.04-2.94$ & 0.34 \\
\hline Previous surgery & 0.94 & $0.21-4.20$ & 0.94 \\
\hline Previous biliary stent & 1.51 & $0.4 I-5.50$ & 0.53 \\
\hline Pain present & 0.84 & $0.34-2.05$ & 0.70 \\
\hline FOLFIRI vs FOLFOX & 0.35 & $0.14-0.87$ & $0.02 *$ \\
\hline PFS $>3$ months with previous NabGem & 0.73 & $0.29-1.85$ & 0.51 \\
\hline$>4$ cycles of Gem-NabP & 0.61 & $0.22-1.73$ & 0.36 \\
\hline
\end{tabular}

Note: *Statistically significant
Table 4 Multiivariate Analysis for OS

\begin{tabular}{|l|l|l|l|}
\hline & HR & $95 \% \mathbf{C l}$ & $P$ \\
\hline No. of metastatic sites $\geq 3$ & 3.01 & $1.85-4.24$ & $0.02 *$ \\
FOLFIRI vs FOLFOX & 0.66 & $0.45-0.0 .72$ & $0.04 *$ \\
\hline
\end{tabular}

Note: *Statistically significant.

patients $(32.2 \%)$, specifically neutropenia was recorded in three patients (19.1\%) as well as diarrhea and nausea/ vomiting. No statistical differences were observed between the treatment regimens. Dose reduction was reported in six (54.5\%) patients for the FOLFOX and in 11 (55\%) patients for the FOLFIRI regimens respectively, no patient stopped or delayed chemotherapy for AEs.

Table 5 Patient Characteristics

\begin{tabular}{|c|c|c|}
\hline & FOLFOX $(\mathbf{N}=I I)$ & FOLFIRI $(\mathbf{N}=20)$ \\
\hline \multicolumn{3}{|l|}{ Neutropenia } \\
\hline GI-2 & $3(27.2 \%)$ & $2(10 \%)$ \\
\hline G3-4 & I (9.1\%) & $2(10 \%)$ \\
\hline \multicolumn{3}{|l|}{ Anemia } \\
\hline GI-2 & $4(36.4 \%)$ & $2(10 \%)$ \\
\hline G3-4 & 0 & 0 \\
\hline \multicolumn{3}{|l|}{ Thrombocytopenia } \\
\hline GI-2 & 0 & I (5\%) \\
\hline G3-4 & 0 & 0 \\
\hline \multicolumn{3}{|l|}{ Diarrhea } \\
\hline GI-2 & I (9.1\%) & $5(40 \%)$ \\
\hline G3-4 & I (9.1\%) & $2(10 \%)$ \\
\hline \multicolumn{3}{|l|}{ Asthenia } \\
\hline GI-2 & $2(18.2 \%)$ & I (5\%) \\
\hline G3-4 & I (9.1\%) & 0 \\
\hline \multicolumn{3}{|l|}{ Nausea/vomiting } \\
\hline $\mathrm{GI}-2$ & $2(18.2 \%)$ & $4(20 \%)$ \\
\hline G3-4 & I (9.1\%) & $2(10 \%)$ \\
\hline
\end{tabular}




\section{Discussion}

PDAC is a leading cause of deaths due to malignancies with a five-year survival pf less than $8 \% .{ }^{9}$ Gemcitabine monotherapy has been the pivot of first-line treatment, but recently, decisive increase in survival has been achieved due to substantial progress in the first-line chemotherapy in locally advanced and metastatic PDAC. Currently, FOLFIRINOX and gemcitabine plus nab-paclitaxel are the two standard regimens recommended in a first-line setting. ${ }^{3,4}$ Many studies have examined the use of secondline therapies after progression to gemcitabine alone in PDAC so far. In the CONKO-003 trial, patients with disease progression after gemcitabine therapy were randomized in two arms: L-OHP plus 5-FU and leucovorin (LV) or 5-FU and LV. A significant benefit in PFS (13 weeks vs nine weeks, $p=0.012$ ) and OS ( 26 weeks versus 13 weeks, $p=0.014$ ) for patients in the oxaliplatin arm have been achieved. ${ }^{10}$ Pelzer et al have reported a better median OS with L-OHP plus 5-FU and folinic acid (OFF) regimen compared with best supportive care alone, whereas the PANCREOX trial recorded a lower median OS with FOLFOX compared with 5-FU monotherapy. ${ }^{11,12}$ The Phase III NAPOLI-1 trial has demonstrated that the liposomal-irinotecan (Nal-IRI) plus 5-FU was effective in patients previously treated with gemcitabine compared with 5-FU monotherapy (median OS of 6.2 months vs 4.2 months; $p=0.012) .{ }^{6}$ Recently, several trials in metastatic cancerthat focused on second-line treatment after progression to FOLFIRINOX and Gem-NabP have been published, although the best second-line chemotherapy sequence is not well defined yet. ${ }^{13}$

In the global Phase III MPACT trial, patients receiving second-line therapy after Gem-NabP have experienced a longer median OS compared with supportive care alone (12.8 vs 6.3 months, respectively) mostly in patients who received fluoropyrimidine-containing second-line regimens (median, 13.5 months). An median OS of 15.7 months in the FOLFIRINOX group compared to 13.7 months in the FOLFOX group has been reported. ${ }^{14}$ Furthermore, another retrospective analysis has confirmed a prolonged median OS of 13.5 months in patients who received fluoropyrimidine-based secondline treatment after first-line Gem-NabP, recording 13.8 months of OS with the FOLFIRINOX, 13.2 with FOLFIRI and 12.8 with FOLFOX/XELOX regimens, with no statistically significant differences among groups. ${ }^{15,16}$ Recently, a retrospective study has reported the clinical benefit of second-line treatment with FOLFOX, FOLFIRI $1 / 3$ or FOLFOXIRI after progression to Gem-NabP regimen, achieving a better median OS in patients receiving FOLFIRI 1/3 (9.7 months) compared with FOLFOX or FOLFIRINOX (3.5 and 6.1 months, respectively). ${ }^{17}$

Alternatively, a prospective multicenter cohort, has shown a significant efficacy and good tolerability of GemNabP after FOLFIRINOX, with a median OS of 8.8 months. ${ }^{18}$ Zaniboni et al have highlighted, in FOLFIRINOX refractory patients, that Gem-NabP with granulocyte colony stimulating factor (GCSF) support could be a potential effective second-line treatment for metastatic pancreatic cancer, supported by a median OS of five months. ${ }^{19}$ In our study patients who received Gem$\mathrm{NaP}$ as first-line, have been treated after progression with FOLFOX or FOLFIRI as second-line. Median PFS was slightly better, but not statistically significant, in the FOLFIRI group than the FOLFOX group (three months vs two months; $p=0.14$ ). However, median OS was statistically better in the FOLFIRI group compared to the FOLFOX group (nine months vs five months; $p=0.01$ ). Moreover, longer median OS in patients with PFS $>3$ months with Gem-NabP was confirmed in the FOLFIRI group compared with the FOLFOX group (nine months vs two months; $p=0.02$ ). To the best of our knowledge, we are not able to explain these results well, therefore, future prospective studies with a large number of patients are awaited to confirm that the PFS $>3$ months with GemNabP may be a predictive factor of efficacy of second-line FOLFIRI compared to FOLFOX.

In our analysis, both regimens have presented good safety profiles, without significant difference between the two groups. No toxic deaths occurred, and most common toxicity were limited to 1-2 grade. Diarrhea and nausea/ vomiting were the most represented toxicities in line with previous studies.

To assess our results, several limitations should be considered, due to the low percentage of patients with metastatic PDAC treated with a second-line therapy, this study was asmall sample size and retrospective study, so it was very difficult to arrange well-balanced patient characteristics, for example, the patients of the FOLFIRI group were younger than the FOLFOX group. And many patients received third-line chemotherapy in the FOLFIRI group. In addition, we have to report that although FOLFOX4 was selected in this study, FOLFOX regimens were some kind of variations as 
FOLFOX6 was also available for patients with pancreatic cancer, however, no comparative study between the two FOLFOX regimens has been performed and future studies are awaited to find the best FOLFOX regimen for patients with metastatic PDAC progressed from first-line Gem-NabP. Nowadays, only a few studies comparing second-line therapies for pancreatic cancer after FOLFIRINOX and Gem-NabP have been published and to our knowledge, limited studies have assessed a comparative evaluation of two fluoropyrimidine-based chemotherapy regimens after Gem-NabP in a real world setting, for these reasons, there is a strong need of randomized, prospective studies with the aim of discovering the best fluoropyrimidine-based chemotherapy regimens after Gem-NabP.

Despite limitations, our results seem to support the use of second-line therapy for patients with metastatic pancreatic cancer and confirm the efficacy and safety of fluoropyrimidine-based combination after Gem-NabP. Our analysis shows a significantly better effectiveness in the FOLFIRI group compared with FOLFOX. Response rates to the second-line regimen and the best safety profile might be influenced by the best response achieved with Gem-NabP in first-line as well as the younger age of patients treated with FOLFIRI. Thus, understanding how the first-line treatment can influence clinical benefit in subsequent treatments is crucial. Overall, the FOLFIRI regimen seemed to allow a better clinical benefit in our patients; a large comparative randomized trial is mandatory to confirm the results.

Finally, additional indications that the second-line choice could be provided by enhanced identification of biologic predictors of the benefit of second-line therapy and specific toxicity investigations.

\section{Data Sharing Statement}

The data used to support the findings of this study are available from the corresponding author upon request.

\section{Ethical Approval}

All procedures performed in studies involving human participants were in accordance with the ethical standards of the institutional and/or national research committee and with the 1964 Declaration of Helsinki and its later amendments or comparable ethical standards. Trial registration number: 14565_oss; Comitato Etico Regionale per la Sperimentazione Clinica della Regione Toscana: Sezione:
AREA VASTA CENTRO; Careggi University of Florence Hospital Ethical Committee.

\section{Informed Consent}

Informed consent was obtained from all individual participants included in the study.

\section{Funding}

There is no funding to report.

\section{Disclosure}

The authors report no conflicts of interest in this work.

\section{References}

1. Maisonneuve P. Epidemiology and burden of pancreatic cancer. Presse Medicale. 2019;48(3):e113-e123. doi:10.1016/j.lpm.2019.02. 030

2. Ferlay J, Colombet M, Soerjomataram I, et al. Cancer incidence and mortality patterns in Europe: estimates for 40 countries and 25 major cancers in 2018. Eur J Cancer. 2018;103:356-387. doi:10.1016/j. ejca.2018.07.005

3. Conroy T, Desseigne F, Ychou M, et al. FOLFIRINOX versus gemcitabine for metastatic pancreatic cancer. $N$ Engl $J$ Med. 2011;19:1819. doi:10.1056/NEJMoa1011923

4. Von Hoff DD, Ervin T, Arena FP, et al. Increased survival in pancreatic cancer with nab-paclitaxel plus gemcitabine. $N$ Engl J Med. 2013;369:1691. doi:10.1056/NEJMoa1304369

5. Sohal DPS, Mangu PB, Khorana AA, et al. Metastatic pancreatic cancer: american Society of Clinical Oncology clinical practice guideline. J Clin Oncol. 2016;34(23):2784-2796. doi:10.1200/ JCO.2016.67.1412

6. Wang-Gillam A, Li CP, Bodoky G, et al. Nanoliposomal irinotecan with fluorouracil and folinic acid in metastatic pancreatic cancer after previous gemcitabine-based therapy (NAPOLI-1): A global, randomised, open-label, Phase 3 trial. Lancet. 2016;545:387. doi:10.1016/ S0140-6736(15)00986-1

7. Eisenhauer EA, Therasse P, Bogaerts J, et al. New response evaluation criteria in solid tumours: revised RECIST guideline (version 1.1). Eur J Cancer. 2009;45:228.

8. National Cancer Institute. Common Terminology Criteria for Adverse Events (CTCAE) Version 4.0. NIH Publ; 2009.

9. Bilimoria KY, Bentrem DJ, Ko CY, et al. Validation of the 6th edition AJCC pancreatic cancer staging system: report from the National Cancer Database. Cancer. 2007;21:1254. doi:10.1002/cncr.22852

10. Pelzer U, Kubica K, Stieler J, et al. A randomized trial in patients with gemcitabine refractory pancreatic cancer. Final results of the CONKO 003 study. J Clin Oncol. 2008;26:4508. doi:10.1200/ jco.2008.26.15_suppl.4508

11. Pelzer U, Schwaner I, Stieler J, et al. Best supportive care (BSC) versus oxaliplatin, folinic acid and 5-fluorouracil (OFF) plus BSC in patients for second-line advanced pancreatic cancer: A Phase III-study from the German CONKO-study group. Eur $J$ Cancer. 2011. doi:10.1016/j.ejca.2011.04.011

12. Crane MM, Clark IBN, Bakker E, Smith S, Swain PS. A microfluidic system for studying ageing and dynamic single-cell responses in budding yeast. PLoS One. 2014. doi:10.1371/journal.pone.0100042

13. Rahma OE, Duffy A, Liewehr DJ, Steinberg SM, Greten TF. Secondline treatment in advanced pancreatic cancer: A comprehensive analysis of published clinical trials. Ann Oncol. 2013. doi:10.1093/ annonc/mdt166 
14. Chiorean EG, Von Hoff DD, Tabernero J, et al. Second-line therapy after nab-paclitaxel plus gemcitabine or after gemcitabine for patients with metastatic pancreatic cancer. Br J Cancer. 2016. doi:10.1038/ bjc. 2016.185

15. Giordano G, Vaccaro V, Lucchini E, et al. Nab-paclitaxel (Nab-P) and gemcitabine $(\mathrm{G})$ as first-line chemotherapy (CT) in advanced pancreatic cancer (APDAC) elderly patients (pts): A "real-life" study. $J$ Clin Oncol. 2015:424.

16. Gourgou-Bourgade S, Bascoul-Mollevi C, Desseigne F, et al. Impact of FOLFIRINOX compared with gemcitabine on quality of life in patients with metastatic pancreatic cancer: results from the PRODIGE 4/ACCORD 11 randomized trial. J Clin Oncol. 2013;23:31. doi:10.1200/JCO.2012.44.4869
17. Point AL, Tougeron D, Pernot S, et al. Three fluoropyrimidine-based regimens in routine clinical practice after nab-paclitaxel plus gemcitabine for metastatic pancreatic cancer: an AGEO multicenter study. Clin Res Hepatol Gastroenterol. 2019;44;229.

18. Sonbol MB, Firwana B, Wang Z, et al. Second-line treatment in patients with pancreatic ductal adenocarcinoma: A meta-analysis. Cancer. 2017;123:1264. doi:10.1002/cncr.30927

19. Zaniboni A, Bertocchi P, Albeni C, et al. Gemcitabine plus nab-paclitaxel as second line and beyond for metastatic pancreatic cancer (MPC): A single institution retrospective analysis. J Clin Oncol. 2014. doi:10.1200/jco.2014.32.15_suppl.e15202

\section{Publish your work in this journal}

Cancer Management and Research is an international, peer-reviewed open access journal focusing on cancer research and the optimal use of preventative and integrated treatment interventions to achieve improved outcomes, enhanced survival and quality of life for the cancer patient.
The manuscript management system is completely online and includes a very quick and fair peer-review system, which is all easy to use. Visit http://www.dovepress.com/testimonials.php to read real quotes from published authors. 